\title{
Tratamento cirúrgico para lipoma submandibular de grande extensão
}

\author{
Surgical treatment for large submandibular lipoma \\ Tratamiento quirúrgico del lipoma submandibular grande
}

Recebido: 25/12/2020 | Revisado: 31/12/2020 | Aceito: 04/01/2021 | Publicado: 06/01/2021

Ricardo Eugenio Varela Ayres de Melo

ORCID: https://orcid.org/0000-0003-1401-457X

Universidade Federal de Pernambuco, Brasil

E-mail: revamelo@yahoo.com

Lohana Maylane Aquino Correia de Lima

ORCID: https://orcid.org/0000-0002-1864-8329

Universidade Federal de Pernambuco, Brasil E-mail: lohanawatson@hotmail.com

Camilla Siqueira de Aguiar

ORCID: https://orcid.org/0000-0001-7376-8393

Universidade Federal de Pernambuco, Brasil

E-mail: camilla.aguiar@outlook.com.br

Rodrigo Henrique Mello Varela Ayres de Melo

ORCID: https://orcid.org/0000-0003-4076-5035 Ministério da Saúde, Brasil

E-mail: rodrigoayres@msn.com

Deise Louise Bohn Rhoden

ORCID: https://orcid.org/0000-0003-4675-5137

Universidade Luterana do Brasil, Brasil

E-mail: deiserhoden17@gmail.com

Milena Mello Varela Ayres de Melo Pinheiro

ORCID: https://orcid.org/0000-0002-5956-1553

Faculdade de Medicina de Olinda, Brasil

E-mail: milena_varela@hotmail.com

José Leonardo de Paiva e Souza

ORCID: https://orcid.org/0000-0002-4033-2099

Universidade Aberta do Terapeuta, Brasil

E-mail: leo.acupuntura@uol.com.br

Neme Portal Bustamante

ORCID: https://orcid.org/0000-0002-5061-9421

Universidad Nacional Federico Villarreal, Peru

E-mail: portal_de_los_implantes@yahoo.com

Juan Carlos Barrenechea Montesinos ORCID: https://orcid.org/0000-0001-6567-9761 Ejército del Perú, Peru

E-mail: jucabacirujano11@hotmail.com

Jussara Diana Varela Ayres de Melo

ORCID: https://orcid.org/0000-0003-0759-6188

Faculdade de Comunicação e Turismo de Olinda, Brasil E-mail: Jussara_varela@hotmail.com

Nely Dulce Varela de Melo Costa Freitas

ORCID: https://orcid.org/0000-0002-2101-5143

Universidade Maurício de Nassau, Brasil

E-mail: nelyvarela@hotmail.com

Jorge Pontual Waked

ORCID: https://orcid.org/0000-0002-2570-0228

Universidade Federal de Campina Grande, Brasil

E-mail: jpwaked@hotmail.com

Arnaldo Caldas de França Júnior

ORCID: https://orcid.org/0000-0002-3713-7532

Universidade Federal de Pernambuco, Brasil

E-mail: caldasjr@alldeia.com.br

Zélia de Albuquerque Seixas

ORCID: https://orcid.org/0000-0001-7843-9134

Universidade Federal de Pernambuco, Brasil

E-mail: zeliaaseixas@gmail.com

Marcela Côrte Real Fernandes

ORCID: https://orcid.org/0000-0002-4160-9985

Universidade Federal de Pernambuco, Brasil

E-mail: marcela.cortereal@gmail.com 


\author{
Victor Leonardo Mello Varela Ayres de Melo \\ ORCID: https://orcid.org/0000-0003-3758-6060 \\ Universidade Federal de Pernambuco, Brasil \\ E-mail: victorlmvamelo@gmail.com \\ Maria Luísa Alves Lins \\ ORCID: https://orcid.org/0000-0002-3957-6117 \\ Universidade Federal de Pernambuco, Brasil \\ E-mail: linsluisam@gmail.com \\ Esdras Marques da Cunha Filho \\ ORCID: https://orcid.org/0000-0002-3214-4741 \\ Faculdade de Medicina de Olinda, Brasil \\ E-mail: esdrasmarquesf@gmail.com \\ Frederico Marcio Varela Ayres de Melo Junior \\ ORCID: https://orcid.org/0000-0001-7377-2392 \\ Universidade Maurício de Nassau, Brasil \\ E-mail: fmvamj31@hotmail.com \\ Bruna Heloísa Costa Varela Ayres de Melo \\ ORCID: https://orcid.org/0000-0002-3158-5131 \\ Centro Universitário Facex, Brasil \\ E-mail: bvmelo98@gmail.com
}

\begin{abstract}
Resumo
Lipoma é um tumor benigno de origem mesenquimal com etiologia incerta, possui predileção por homens, entre os 50 e 70 anos de idade, sendo a região de tronco e extremidades os sítios mais comuns. Clinicamente, apresenta-se como lesão bem circunscrita, móvel, macia à palpação, indolor e de base séssil ou pedunculada. O diagnóstico é majoritariamente clínico com a confirmação por meio de análise histopatológica e por exames de imagens, ao qual o tratamento de escolha é a ressecção cirúrgica conservadora. Este trabalho tem por objetivo relatar o caso de tratamento cirúrgico de lipoma extenso na região submandibular em uma paciente do sexo feminino, 67 anos de idade, que apresentava aumento de volume na região submandibular direita. Ao exame clínico, apresentou lesão bem delimitada, móvel, macia à palpação e indolor. Foi preconizada a realização de uma biopsia excisional. Sob anestesia geral, realizou-se a incisão submandibular de Risdon, divulsão dos tecidos subcutâneos e hemostasia dos vasos sangrantes. A cirurgia seguiu com a ressecção da lesão, toalete da cavidade e sutura por planos. A peça patológica foi encaminhada para o Serviço de Anatomopatologia do Hospital das Clinicas da Universidade Federal de Pernambuco, medindo $14 \mathrm{~cm} \times 6,5 \mathrm{~cm}$, com confirmação da hipótese de Lipoma. O caso foi acompanhado por seis anos apresentando bom resultado estético e funcional sem recidiva. Portanto, mesmo que não muito frequentes na região de cabeça e pescoço, cabe ao Cirurgião Buco Maxilo Facial conhecer as características desse tipo de patologia, bem como a forma adequada de tratamento para prevenir recidiva.
\end{abstract}

Palavras-chave: Lipoma; Neoplasias bucais; Procedimentos cirúrgicos operatórios.

\begin{abstract}
Lipoma is a benign tumor of mesenchymal origin with uncertain etiology. They have a predilection for men, between 50 and 70 years old and the trunk and extremities being the most common sites. Clinically, they appear as wellcircumscribed lesions, mobile, soft to the touch, painless with a sessile or pedunculate base. The diagnosis is mostly clinical being confirmed through histopathological analysis and imaging exams and the treatment of choice is conservative surgical resection. The aim of this paper is to report a case of surgical treatment of an extensive lipoma in the submandibular region in a woman patient, 67 years old, who presented an increase in volume in the right submandibular region. On clinical examination, the patient presented a well-defined, mobile, soft palpation and painless lesion. An excisional biopsy was recommended. Under general anesthesia, Risdon's submandibular incision, divulsion of subcutaneous tissues and hemostasis of bleeding vessels were performed. The surgery continued with resection of the lesion, cavity toilet and continuous intradermal suture using layers. The pathological specimen was sent to the Pathology Department of the Hospital das Clínicas, Federal University of Pernambuco which showed a lesion measuring $14 \mathrm{~cm} \times 6.5 \mathrm{~cm}$ in size with obtaining confirmation of lipoma hypothesis. The case was accompanied for six years showing good aesthetic and functional results without recurrence. Therefore, even if not very frequent in the head and neck region, the Maxillofacial Surgeon needs to know the characteristics of this type of pathology, as well as the appropriate form of treatment to prevent recurrences.
\end{abstract}

Keywords: Lipoma; Mouth neoplasms; Surgical procedures, Operative.

\title{
Resumen
}

El lipoma es un tumor benigno de origen mesenquimatoso de etiología incierta, tiene predilección por el hombre, entre los 50 y 70 años, siendo el tronco y las extremidades las localizaciones más frecuentes. Clínicamente se presenta como una lesión bien circunscrita, móvil, suave al tacto, indolora y de base sésil o pediculada. El diagnóstico es mayoritariamente clínico con confirmación mediante análisis histopatológico y mediante exámenes de imagen, para lo que el tratamiento de elección es la resección quirúrgica conservadora. Este estudio tiene como objetivo reportar el caso de tratamiento quirúrgico de lipoma extenso en región submandibular en una paciente de 67 años, que presentó aumento de volumen en región submandibular derecha. En el examen clínico la lesión estaba bien definida, móvil, 
suave al tacto e indolora. Se recomendó una biopsia por escisión. Bajo anestesia general se realizó incisión submandibular de Risdon, divulgación de tejidos subcutáneos y hemostasia de vasos sangrantes. La cirugía continuó con la resección de la lesión, sanado de la cavidad y sutura en capas. La pieza patológica fue enviada al Servicio de Anatomopatología del Hospital das Clínicas, Universidade Federal de Pernambuco, de 14 x 6,5 cm, con confirmación de la hipótesis de Lipoma. El caso fue seguido durante seis años, mostrando buenos resultados estéticos y funcionales sin recurrencia. Por tanto, aunque no sea muy frecuente en la región de cabeza y cuello, le corresponde al Cirujano Oral Maxilofacial conocer las características de este tipo de patologías, así como la forma de tratamiento adecuada para prevenir la recurrencia.

Palabras clave: Lipoma; Neoplasias de la boca; Procedimientos quirúrgicos operativos.

\section{Introdução}

Lipoma é um tumor benigno, de origem mesenquimal, composto por tecido de gordura. A sua patogênese ainda é desconhecida e embora ele apareça mais comumente em pessoas obesas, sabe-se que o seu metabolismo é totalmente independente da gordura corporal. É mais comum na região de tronco e extremidades do corpo com menor prevalência na região de cabeça e pescoço (Neville, Damm, Allen \& Chin, 2016; Won, Hur, Ohn \& Mun, 2020), apresenta predileção pelo sexo masculino, com incidência entre 50 e 70 anos de idade (Osterne, Lima- Verde, Turatti, Nanaka \& Cavalcante, 2019).

Clinicamente, os lipomas podem possuir variação, pois dependerá do tamanho, localização e crescimento da lesão. São principalmente indolores e por este fato, os pacientes só procuram atendimento por questões estéticas ou quando sentem compressão de alguma estrutura anatômica o que acarretaria em sintomatologia dolorosa (Chatterjee, Prasad, Nayak \& Mahato, 2015; Najaf, Catier, Favier \& Garrel, 2019). As lesões apresentam superfície lisa, são móveis e macios à palpação, podem ser sésseis ou pedunculadas com coloração amarelada nas lesões mais superficiais e rosada nas mais profundas (Lazano-Masdermon, Franco-Muñoz, Cortina de la Calle, Flores-Terry, 2017; Mohan, Aggarwal, A., \& Aggarwal, S., 2016). Em relação à profundidade podem estar localizadas na camada gordurosa ou sub muscular, sendo a camada gordurosa mais prevalente em 75\% dos casos. Além disso, os lipomas crescem lentamente (Derin \& Yaprak, 2018) e possuem um tamanho médio de $3 \mathrm{~cm}$, sendo considerados lipomas gigantes os que exibem um tamanho superior a $5 \mathrm{~cm}$ (Osterne et al., 2019; Kim, Chai, Lee \& Park, 2018; Sing, V., Kumar \& Sing, A., 2017).

Os lipomas de cabeça e pescoço tem características específicas para a sua abordagem devido à complexidade anatômica da região, pois eles podem se desenvolver em zonas de perigo da face nas quais envolvem veias, artérias e nervos importantes que podem ser lesionados durante a remoção (Kim et al., 2018).

Geralmente as suspeitas clínicas são suficientes para caracterizar a lesão, principalmente as localizadas no tecido subcutâneo, sendo necessário em poucos casos a utilização de exames adicionais (Abajo, Polo, Barrena \& Poveda, 2016). Dentro dos exames complementares para o diagnóstico, podem ser solicitados exames de imagem que incluem ultrassonografia, tomografia computadorizada e ressonância magnética. As lesões se apresentam bem circunscritas com características de imagem ecogênica, hipodensa e com alta intensidade de sinal de gordura, respectivamente (Burt \& Muang, 2017).

Histologicamente, os lipomas podem ser classificados em lipomas simples e variantes como fibrolipoma, angiolipoma, osteolipoma, condrolipoma, miolipoma, lipoma de células fusiformes, lipoma pleomórfico e lipoma intramuscular (Osterne et al., 2019). O lipoma clássico é composto por adipócitos maduros, bem circunscritos, que podem se apresentar em arranjo lobular e possuir uma fina cápsula de tecido fibroso. A variante mais frequente é o fibrolipoma, caracterizado por tecido adiposo em arranjo lobular, separado por grande quantidade de tecido fibroso. A ocorrência das outras variantes é rara (Neville et al., 2016; Mungul, Maharaj \& Masege, 2017; Harfuch et al., 2020), assim como a transformação maligna em lipossarcoma (Abajo et al., 2016).

O tratamento de escolha para os lipomas é a excisão cirúrgica conservadora, porém métodos de remoção alternativos como a lipoaspiração e a remoção a laser tem sido descrito na literatura com o objetivo de serem mais estéticos e diminuir as 
cicatrizes (Boyer, Monette, Nguyen, Zipp, Aughenbaugh \& Nimunkar, 2015; Castanha, Xavier, Guedes, Lyra \& Coutinho, 2020). Nesse contexto, o manejo terapêutico cirúrgico dos lipomas podem ser variáveis e devem ser levados em consideração a localização, tamanho da lesão, a necessidade de exames complementares, o estado de saúde do paciente e a experiência do cirurgião, orientando a abordagem cirúrgica de escolha para ter uma menor comorbidade pós- operatória (Najaf et al., 2019). A maioria das variantes microscópicas não afeta o prognóstico que é favorável e a recidiva é incomum (Neville et al., 2016).

\section{Metodologia}

Este artigo trata-se de um relato de caso clínico com abordagem descritiva, qualitativa, ao qual o pesquisador é instrumento indispensável. (Pereira et al., 2018). O registro foi conduzido em total concordância com os princípios éticos de acordo com a declaração de Helsinque, revisada em 2013. A paciente concordou com a divulgação de dados e fotografias através da assinatura do Termo de Consentimento Livre e Esclarecido, ficando claro que as informações seriam utilizadas exclusivamente com o propósito de divulgação científica. Os dados da paciente foram avaliados após diagnóstico e a paciente acompanhada por 06 anos para verificar a efetividade do tratamento. Realizou-se uma revisão da literatura utilizando-se as bases de dados PubMed e Scielo com restrição temporal entre os anos de 2015 a 2020.

\section{Relato de Caso}

Paciente do sexo feminino, 67 anos de idade, compareceu no Ambulatório de Cirurgia e Traumatologia Buco Maxilo Facial do Curso de Odontologia da Universidade Federal de Pernambuco queixando-se de aumento de volume na região submandibular direita, com aproximadamente 5 anos de evolução.

Ao exame clínico, apresentava uma lesão bem delimitada com consistência macia, móvel e indolor à palpação. A paciente era saudável e não apresentava antecedentes relevantes, além do sobrepeso. Com as características clínicas sugestivas de lipoma, solicitou-se exames por imagem e através de ultrassonografia apresentou uma lesão encapsulada com imagem hipoecogênica e bem delimitada corroborando com as indicações clínicas. Baseado nos achados, optou-se por realizar uma biópsia do tipo incisional, confirmando a hipótese de diagnóstico (Figuras 1A e 1B).

Figuras 1. Aspecto pré-operatório - (A) vista lateral direita; (B) Vista ântero - inferior.
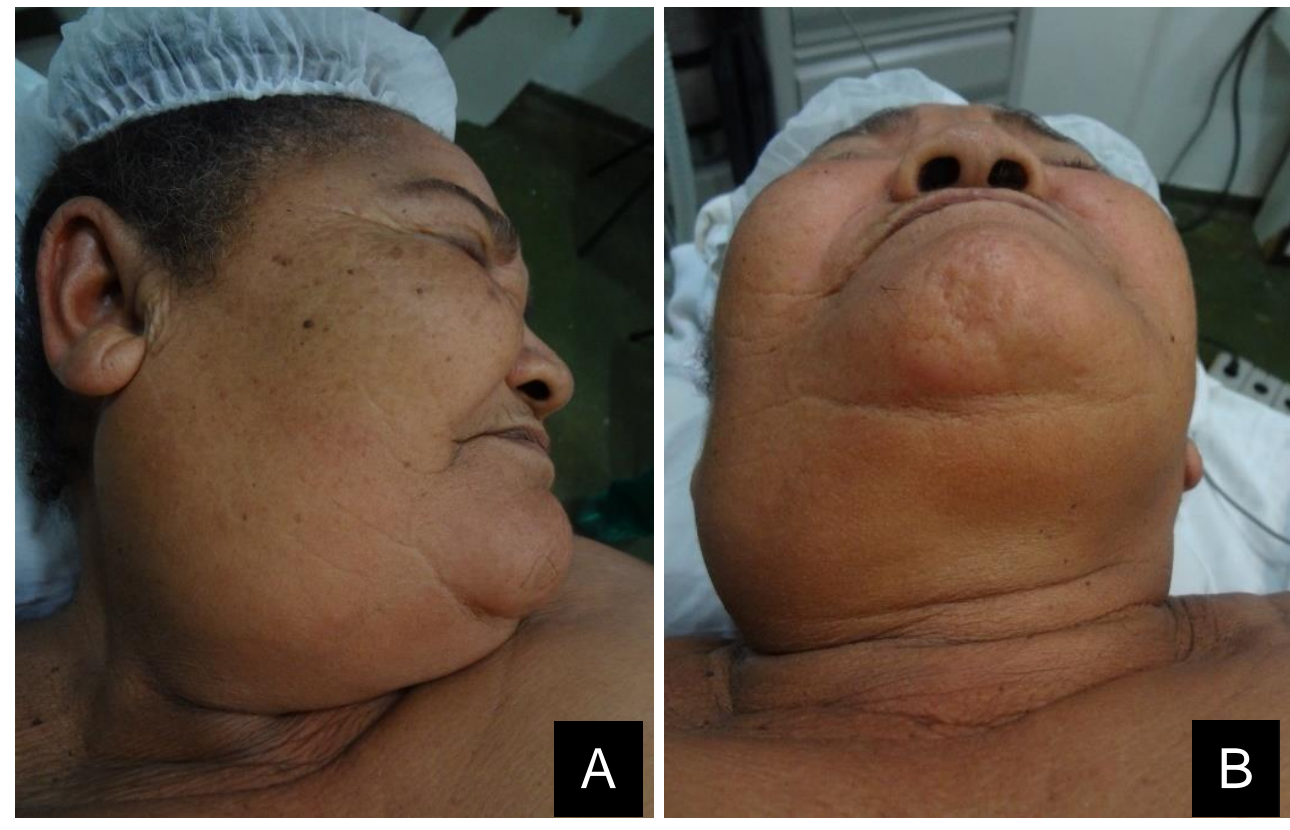

Fonte: Autores. 
Na Figura 1 demonstra aumento de volume na região submandibular direita, bem delimitada, móvel e macia à palpação com 05 anos de evolução.

Sob anestesia geral, foi preconizado o acesso submandibular de Risdon para isso, utilizou-se como orientação a demarcação de quatro pontos de referência para a incisão: O primeiro ponto no ângulo da mandíbula; o segundo ponto na borda posterior do ramo ascendente da mandíbula; o terceiro ponto na basilar mandibular e o quarto ponto na borda anterior do músculo esternocleidomastoideo. Feita a união desses pontos, localizou-se o triângulo de Farabeuf ao qual, realizada uma bissetriz, temos a presença de dois triângulos, sendo um superior e outro inferior. No triângulo superior traça-se uma nova bissetriz e então temos a localização da incisão de Risdon que foi realizada, paralela à basilar mandibular, através dos tecidos cutâneos e subcutâneos com divulsão até o músculo platisma (Figuras 2 e 3).

Figura 2. Transoperatório.

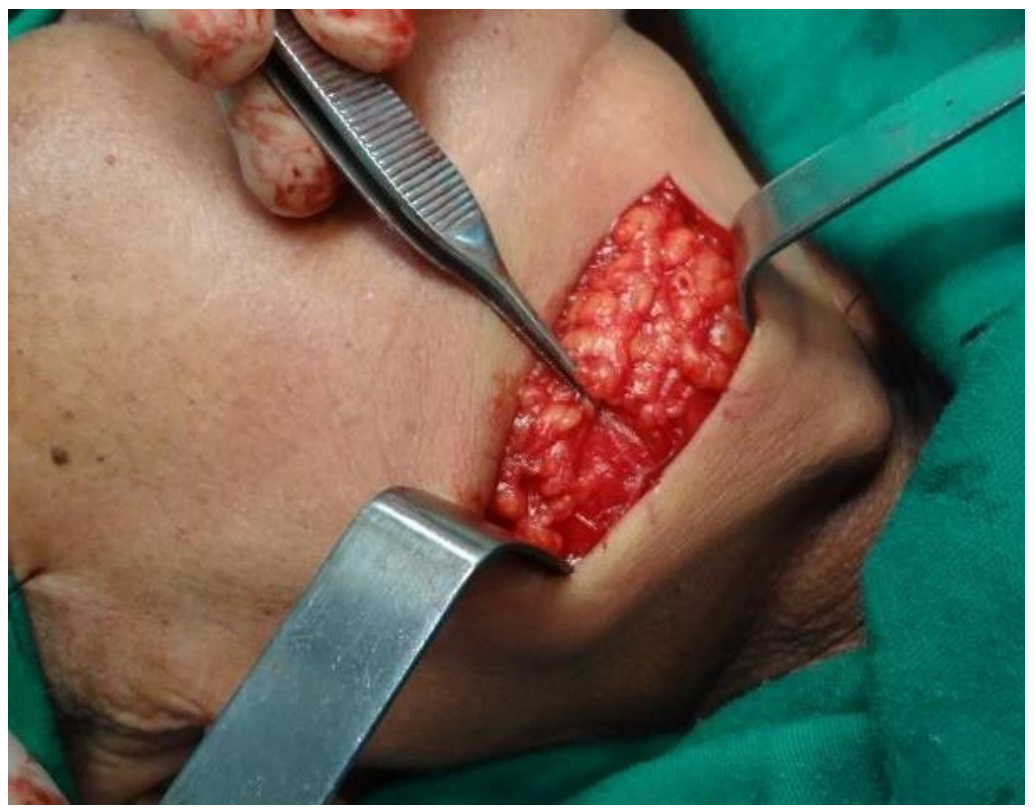

Fonte: Autores.

Na Figura 2 é possível visualizar a incisão de Risdon e a exposição do músculo platisma.

Figura 3. Identificação do músculo platisma.

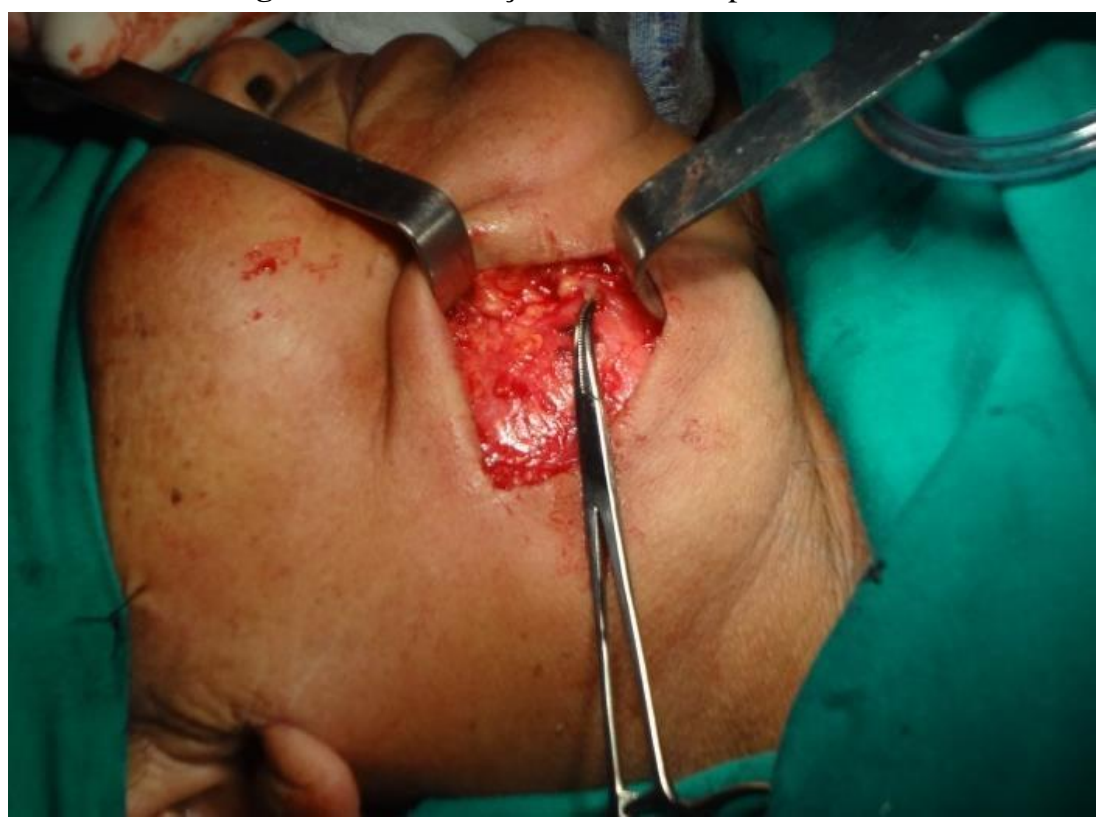




\section{Fonte: Autores.}

Em sequência, realizou-se a diferenciação do músculo platisma como pode ser observado na Figura 3, utilizando-se de duas pinças hemostáticas do tipo Crile curva para preensão interna ao músculo, que servem de orientação da incisão, seguindo de identificação do músculo para posterior reposicionamento para evitar lesionar o ramo mandibular do sétimo par craniano (Ramo de Jaffé).

Com a região exposta foi possível visualizar a lesão ao qual acometia a glândula parótida, realizou-se então a hemostasia dos ramos cervicais da artéria facial (ramo submentoniano, palatino descendente, glandular e tonsilar) e ressecção total da lesão (Figuras 4, 5, 6 e 7). A cirurgia seguiu com toalete da cavidade e suturas a pontos separados por planos musculares e sutura do tipo intradérmica continua em plano cutâneo com fio Prolene cardiovascular 5.0.

Figura 4. Diferenciação do músculo platisma demonstrando a exposição da lesão.

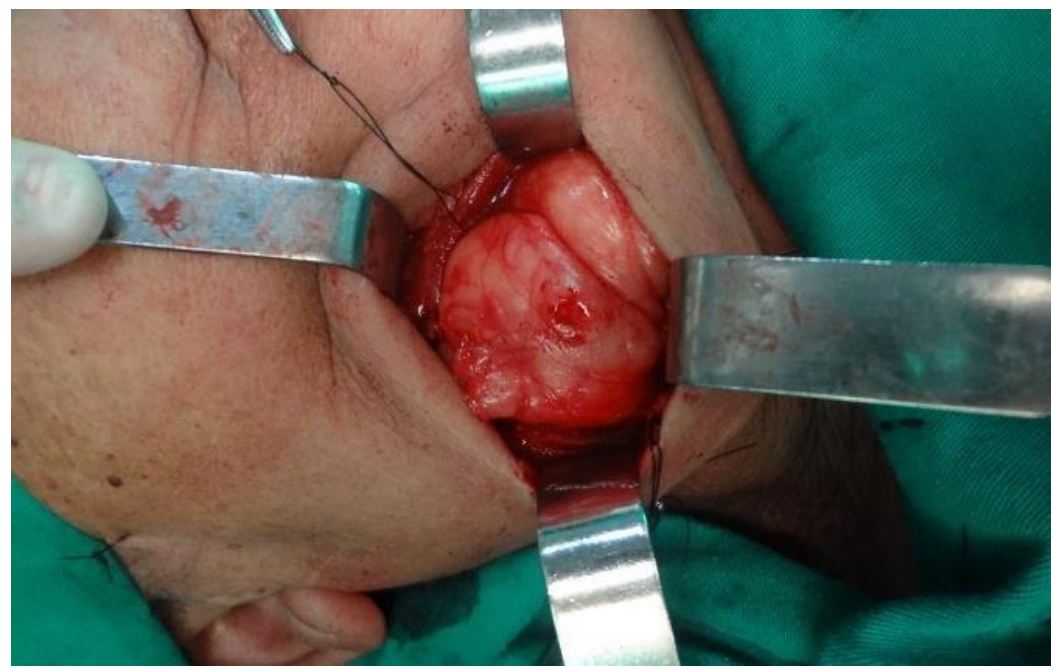

Fonte: Autores.

Figura 5. Exposição da lesão com hemostasia dos vasos sangrantes.

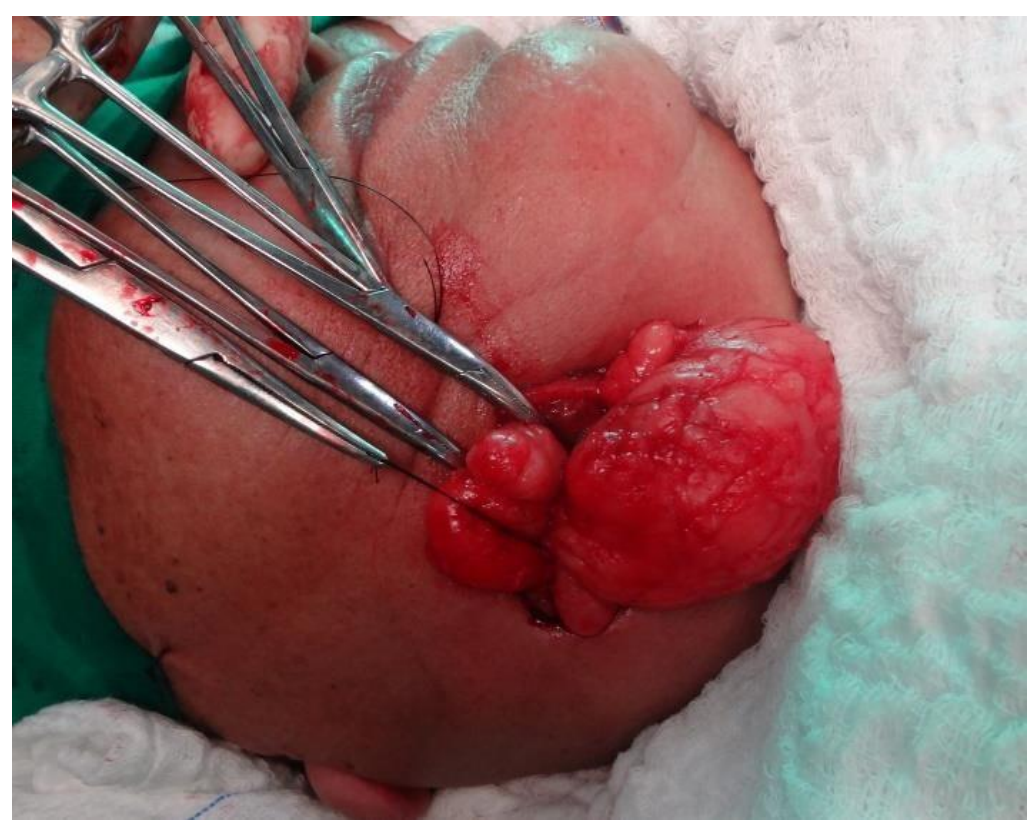

Fonte: Autores. 
Figura 6. Transoperatório. Ressecção da lesão.

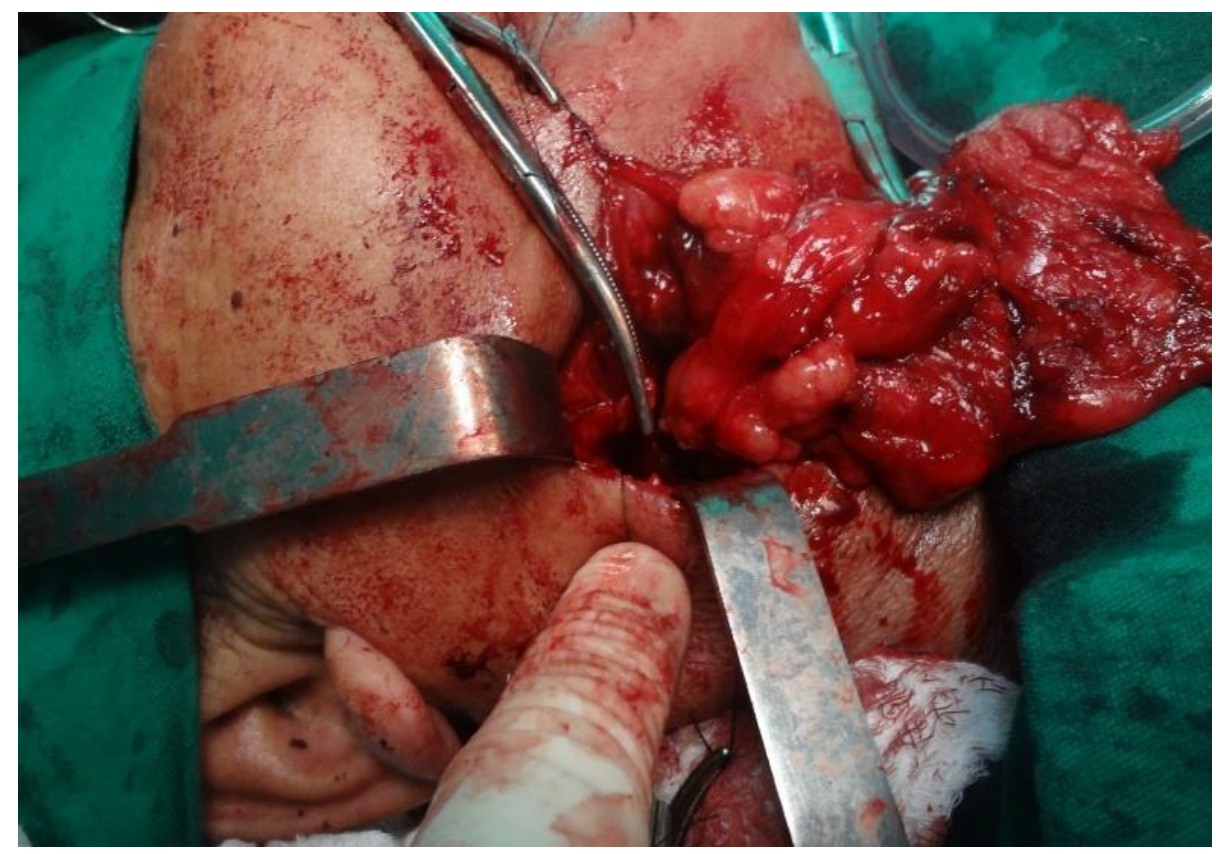

Fonte: Autores.

Na Figura 6 observa-se o pinçamento e laqueadura dos ramos cervicais da artéria facial (ramo submentoniano, palatino descendente, glandular e tonsilar).

Figura 7. Transoperatório. Ressecção da lesão de grande extensão na sua porção cervical.

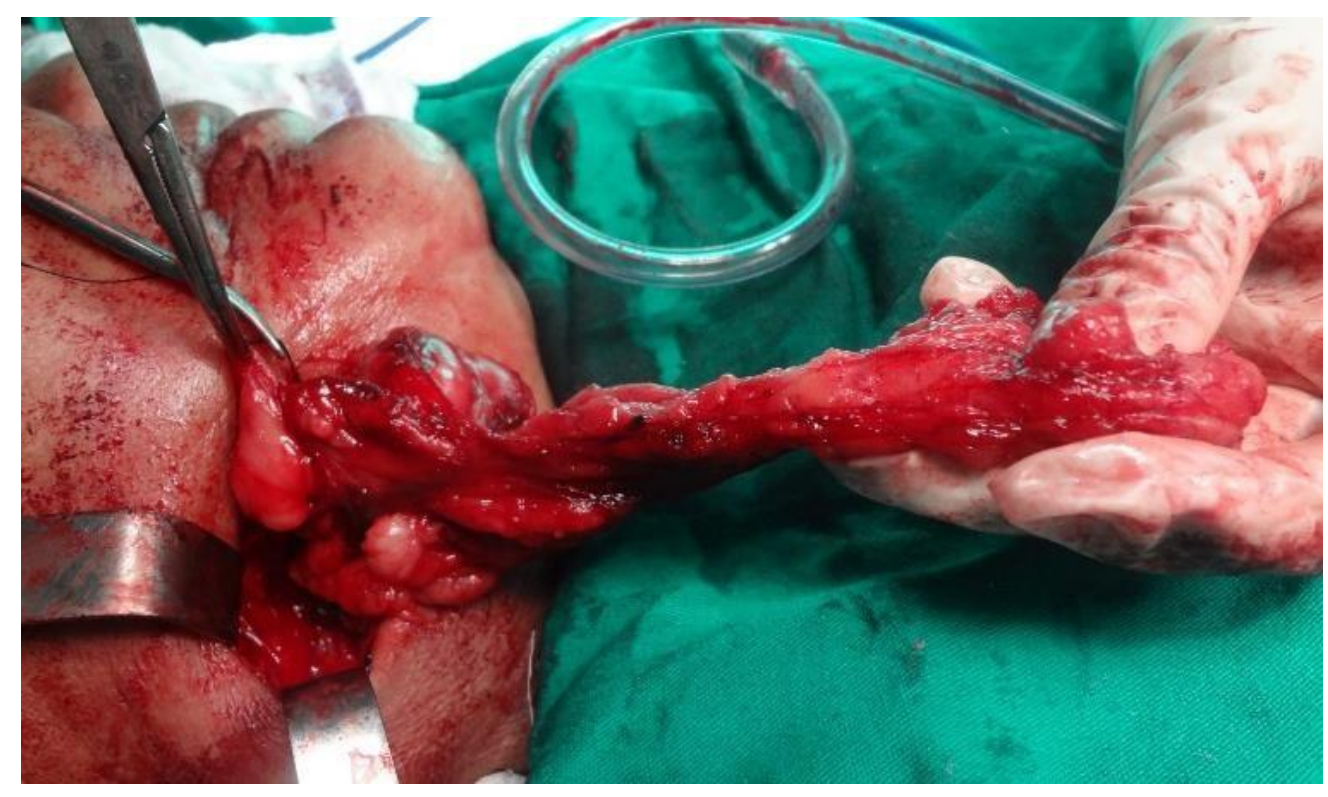

Fonte: Autores.

No pós-operatório foi prescrito para a paciente: acompanhamento dos sinais vitais; dieta líquida pastosa, hiperproteica e hipercalórica de 02 em 02 horas; soro glicosado a 5\%, 1500ml, 21 gotas/minuto, contínuo; complexo B + vitamina C, 01 ampola em cada fase; cefalotina sódica 1g, 01 frasco-ampola e Dexametasona 4mg, 01 frasco-ampola (2,5ml=10mg) intravenoso 06 em 06 horas; cloridrato de metoclopramida 10mg, 01 ampola intravenoso de 06 em 06 horas, em casos de 
náuseas ou vômitos; paracetamol 500mg associado à fosfato de codeína 30mg, 01 comprimido via oral de 08 em 08 horas, nas primeiras 24 horas. Crioterapia contínua naregião nas primeiras 24 horas e substituição por termoterapia continua ao segundo diapós-operatório. Para realização dos curativos, foi prescrito pomada dermatológica de fibrinolisina + desoxirribonuclease + cloranfenicol, 02 vezes ao dia sob o ferimento.

A peça patológica foi encaminhada para o Serviço de Anatomopatologia do Hospital das Clinicas da Universidade Federal de Pernambuco que mostrou uma lesão macroscópica com coloração amarelada medindo $14 \mathrm{~cm} \times$ x,5cm de tamanho. Ao exame microscópico, caracterizava-se por grupamento de adipócitos maduros, agrupados em arranjo lobular, entremeados por feixes de tecido conjuntivo com obtenção da confirmação da hipótese de Lipoma (Figura 8).

Figura 8. Lâmina histopatológica.

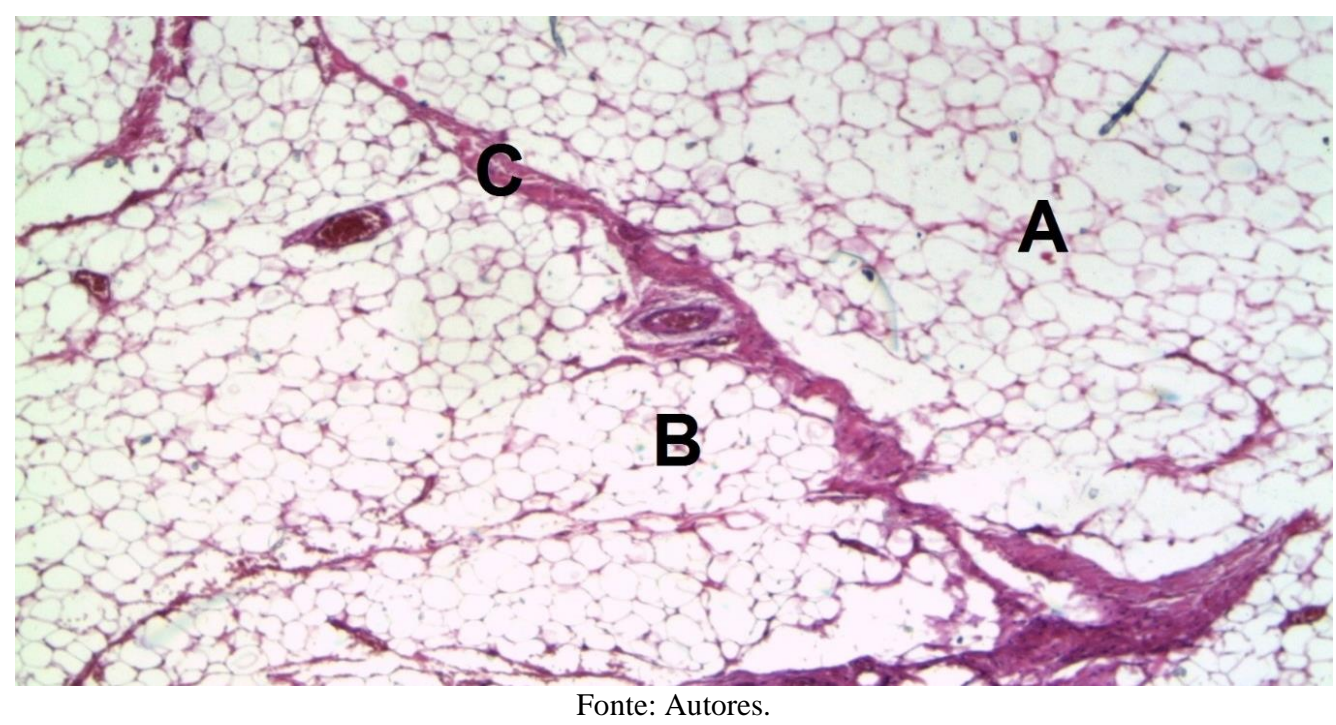

Na Figura 8 da lâmina histopatológica demonstra grupamentos de adipócitos maduros (A) em arranjo lobular (B), entremeados por feixes de tecido conjuntivo frouxo (C) (H.E.X40).

A paciente recebeu alta hospitalar no quinto dia de pós-operatório sob os cuidados descritos. Retornou com 07 dias ao Ambulatório de Cirurgia e Traumatologia Buco Maxilo Facial da Universidade Federal de Pernambuco para acompanhamento sem sinais flogísticos e com 15 dias para remoção da sutura. O controle pós-operatório seguiu com 30, 60, 90, 120 dias e anualmente ao qual apresentava boa cicatrização com estética favorável e preservação da função local, demonstrando que não houve nenhum comprometimento nervoso. O caso foi proservado por 6 anos, sem evidências de recidiva (Figuras 9A e 9B). 
Figuras 9 A e B. Pós-operatório com 06 anos de evolução da paciente.

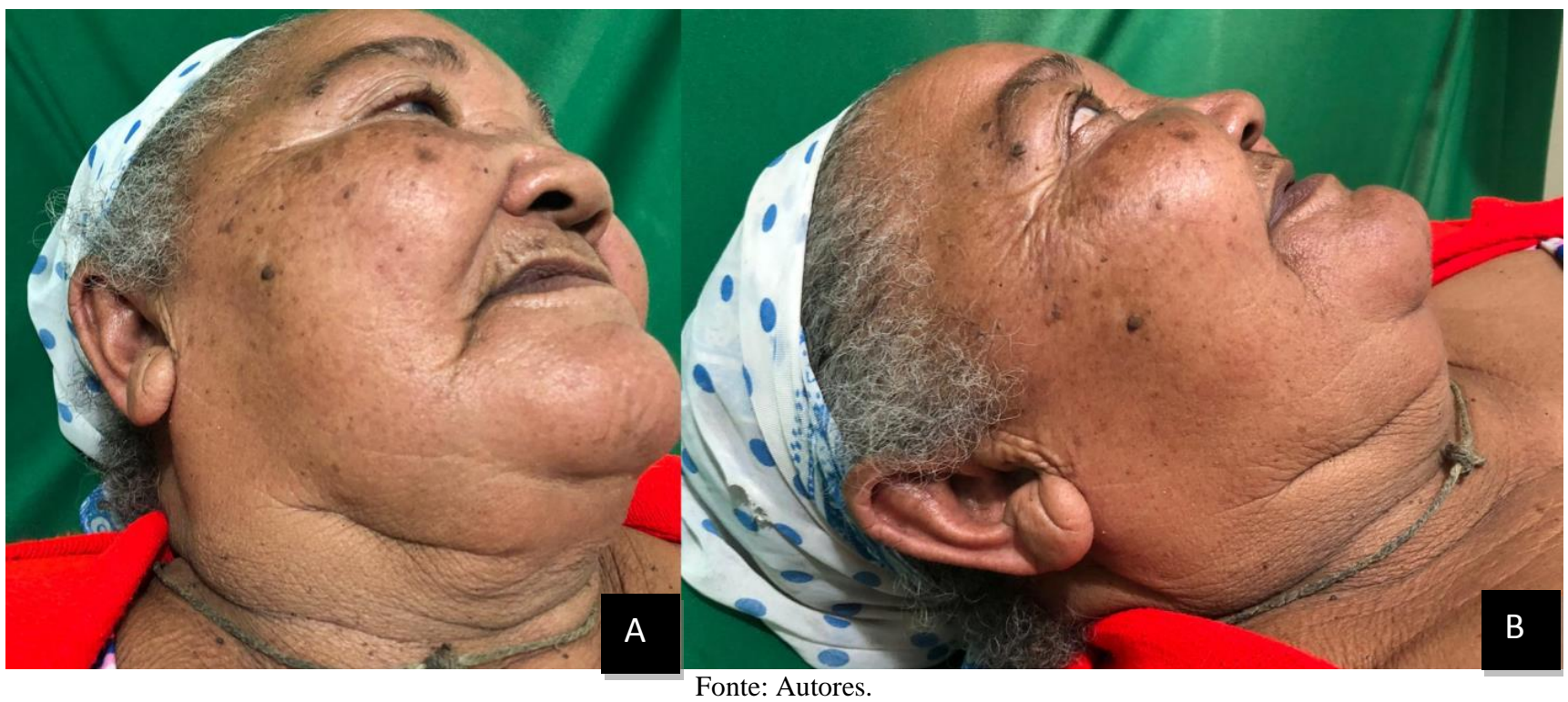

\section{Discussão}

Este artigo relata o tratamento cirúrgico de uma paciente que apresentou um lipoma de grande extensão na região submandibular ao qual segundo Neville et al. (2016) e Won et al. (2020) é um local de menor prevalência, pois na literatura essas lesões acometem com maior frequência a região de tronco e extremidades do corpo.

Possuem predileção por pessoas do sexo masculino, entre 50 e 70 anos de idade (Osterne et al., 2019; Kim et al., 2018). As lesões apresentam um tamanho médio de $3 \mathrm{~cm}$ e são considerados lipomas de grandes proporções quando exibem um tamanho superior a $5 \mathrm{~cm}$. Em relação a profundidade podem estar localizados na camada gordurosa ou sub muscular, sendo a camada gordurosa mais prevalente em 75\% dos casos (Osterne et al., 2019; Derin \& Yaprak, 2017; Kim et al., Singh et al., 2017). A paciente tinha 67 anos de idade, contudo o caso clínico relatado contrapõe-se à literatura pois é do sexo feminino. Além disso, saindo da média descrita, o lipoma média $14 \mathrm{~cm}$ x 6,5cm de tamanho, sendo assim considerado de grande extensão com localização na região submandibular o que é menos comum.

Clinicamente, os lipomas apresentam crescimento lento, são indolores com superfície lisa, móveis e macios à palpação. Podem apresentar uma base séssil ou pedunculada comcoloração amarelada nas lesões mais superficiais e rosada nas mais profundas (Chatterjee et al., 2015; Najaf et al., 2019; Lazano-Masdemon et al., 2017; Mohan et al., 2016). A paciente procurou atendimento queixando-se do aumento de volume na região submandibular direita, com evolução de 05 anos. A lesão era bem delimitada, com superfície lisa, móvel, macio a palpação e coloração rosada, o que corrobora com a literatura descrita.

Os lipomas de cabeça e pescoço tem características específicas devido à complexidade anatômica da região, pois eles podem se desenvolver em zonas de perigo da face nas quais podem envolver veias, artérias e nervos importantes que podem ser lesionados durante a remoção (Kim et al., 2018). No caso relatado, foi utilizado da demarcação de pontos de orientação para evitar lesionar o ramo mandibular do nervo facial que se dirige anterior na glândula parótida, em direção cervicofacial, passando profundamente ao músculo platisma, sendo uma importante estrutura anatômica que poderia causar perda da função dos movimentos faciais da paciente. Durante o procedimento cirúrgico, vasos cervicais da artéria facial foram seccionados por estarem relacionados com a lesão.

Segundo Abajo et al. (2016) e Burt e Huang (2017) as suspeitas clínicas, geralmente são suficientes para estabelecer uma hipótese de diagnóstico, porém exames complementares podem ser solicitados. Com as características clínicas sugestivas 
de lipoma, solicitamos uma ultrassonografia da região cervical para complementar a hipótese que mostrou imagem encapsulada, hipoecogênica e bem delimitada corroborando com os achados clínicos.

Histopatologicamente, o caso apresentado corroborou com Mungul et al. (2017), Neville et al. (2016), Osterne et al. (2019) e Harfuch et al. (2020), pois ao exame histopatológico a lesão se apresentava com grupamento de adipócitos maduros, bem circunscrito em arranjo lobular entremeado com feixes de tecido conjuntivo frouxo caracterizando a forma clássica de lipoma.

No nosso caso optou-se pela ressecção cirúrgica conservadora o que promoveu um excelente resultado estético, embora métodos alternativos tenham sido descritos na literatura como a lipoaspiração e a remoção a laser com o objetivo de serem mais estéticos (Boyer et al., 2015). Segundo Castanha et al. (2020), Najaf et al. (2016) e Neville et al. (2016) devem ser levados em considerações fatores como a localização, tamanho da lesão, estado de saúde da paciente e experiencia do cirurgião para orientar a abordagem cirúrgica, assim como descrevemos nesse caso ao qual foi proservado por dois anos e não apresentou recidiva da lesão sendo, portanto, um prognóstico favorável.

\section{Conclusão}

Os lipomas são tumores benignos que apesar de não acometerem com frequência as regiões da cabeça e pescoço, devem ser levados em consideração em pacientes que apresentem aumento de volume na região submandibular, bem delimitado, macio à palpação e indolor. O tratamento de nossa escolha é a ressecção cirúrgica total da lesão por apresentar um prognóstico favorável e evitar recidivas. O acesso submandibular de Risdon demonstrou-se como boa opção para lesões na região cervical, pois quando realizado de maneira adequada, evita lesões a estruturas anatômicas importantes da região, além de apresentar um resultado estético satisfatório.

\section{Referências}

Abajo, B. M., Polo, J. R. T., Barrena, I. R., \& Poveda, M. D. (2016). Lipomas: classification, dermatologic manifestations and treatment. International Journal of Cancer Research and Prevention, 9(2), 125.

Boyer, M., Monette, S., Nguyen, A., Zipp, T., Aughenbaugh, W. D., \& Nimunkar, A. J. (2015). A review of techniques and procedures for lipoma treatment. Clinic Dermatol, 3(4), 105-112.

Burt, A. M., \& Huang, B. K. (2017). Imaging review of lipomatous musculoskeletal lesions. Sicot-j, 3(34), 1-17.

Castanha, D. M., Xavier, E. C. N., Guedes, R. L., Lyra, T. C., Coutinho, M. A. C. R., Brasil Junior, O. (2020). Tratamento cirúrgico de lipoma de grandes proporções em região submandibular: relato de caso. Research, Society and Development, 9(10), 1-12.

Chatterjee, S., Prasad, S., Nayak, S. D., \& Mahato, S. P. (2015). Anterior neck lipoma - A giant predicament. Hellenic Journal of Surgery, 87(2), $203-205$.

Derin, A. T., \& Yaprak, N. (2017). Lipomas: Review and evaluation of the literature. Clin Surg, 2, 1615.

Harfuch Capdevil, T., Vinitzky Brener, I., Liceaga Escalera, C. J., Aldape Barrios, B. C., \& Rivero, A. (2020). Oral lipoma: Review of literature and case report. Odontología Vital. (32), 79-86.

Kim, J. S., Choi, M. S., Lee, S. J., \& Park, B. C. (2018). Clinico-surgical characteristics of head and neck lipomas. Dermatologic Surgery, 44(6), 893-895.

Lozano-Masdemon, B., Franco-Muñoz, M., Cortina de la Calle, P., \& Flores-Terry, M. Á. (2017). Lipoma móvil encapsulado. Arch. argent. pediatr, 115(1), 99-100.

Mohan, A., Aggarwal, A., \& Aggarwal, S. (2016). Intraoral Lipoma: Rare Case report with clinical and histopathological study. Journal of Pierre Fauchard Academy (India Section), 30(1), 32-34.

Mungul, S., Maharaj, S., \& Masege, S. D. (2017). Lingual Fibrolipoma-A rare clinicopathological entity. South African Journal of Surgery, 55(2), 36-38.

Najaf, Y., Cartier, C., Favier, V., \& Garrel, R. (2019). Symptomatic head and neck lipomas. European annals of otorhinolaryngology, head and neck diseases, 136(2), 127-129.

Neville, B. W., Damm, D. D., Allen, C. M., \& Chi, A. C. (2016). Oral and Maxillofacial Pathology. Elsevier Health Sciences.

Osterne, R. L., Lima-Verde, R., Turatti, E., Nanaka, C. F. W., \& Cavalcante, R. B. (2019). Oral cavity lipoma: a study of 101 cases in a Brazilian population. Jornal Brasileiro de Patologia e Medicina Laboratorial, 55(2), 148 - 159. 
Research, Society and Development, v. 10, n. 1, e16110111488, 2021

(CC BY 4.0) | ISSN 2525-3409 | DOI: http://dx.doi.org/10.33448/rsd-v10i1.11488

Pereira A. S. et al. (2018). Metodologia da pesquisa científica. Ed. UAB/NTE/UFSM. https://repositorio.ufsm.br/bitstream/handle/1/15824/Lic_Computacao_Metodologia-Pesquisa-Cientifica.pdf?sequence=1.

Singh, V., Kumar, V., \& Singh, A. K. (2017). Giant Palmar Lipoma: A Rare Presentation. World journal of plastic surgery, 6(2), 248.

Won, J. H., Hur, K., Ohn, J., \& Mun, J. H. (2020). Surgical management of lipomas: Proposal of the Z-incision design and surgical algorithm based on tumor size. Dermatologic Therapy, 33(1), e13151. 